\title{
Energy Distribution of a Regular Black Hole Solution in Einstein-Nonlinear Electrodynamics
}

\author{
I. Radinschi, ${ }^{1}$ F. Rahaman, ${ }^{2}$ Th. Grammenos, ${ }^{3}$ A. Spanou, ${ }^{4}$ and Sayeedul Islam ${ }^{2}$ \\ ${ }^{1}$ Department of Physics, "Gheorghe Asachi” Technical University, 700050 Iasi, Romania \\ ${ }^{2}$ Department of Mathematics, Jadavpur University, Kolkata 700 032, India \\ ${ }^{3}$ Department of Civil Engineering, University of Thessaly, 38334 Volos, Greece \\ ${ }^{4}$ School of Applied Mathematics and Physical Sciences, National Technical University of Athens, 15780 Athens, Greece
}

Correspondence should be addressed to F. Rahaman; rahaman@iucaa.ernet.in

Received 23 February 2015; Revised 11 May 2015; Accepted 25 May 2015

Academic Editor: Giorgio Kaniadakis

Copyright ( 2015 I. Radinschi et al. This is an open access article distributed under the Creative Commons Attribution License, which permits unrestricted use, distribution, and reproduction in any medium, provided the original work is properly cited.

\begin{abstract}
A study about the energy momentum of a new four-dimensional spherically symmetric, static and charged, regular black hole solution developed in the context of general relativity coupled to nonlinear electrodynamics is presented. Asymptotically, this new black hole solution behaves as the Reissner-Nordström solution only for the particular value $\mu=4$, where $\mu$ is a positive integer parameter appearing in the mass function of the solution. The calculations are performed by use of the Einstein, Landau-Lifshitz, Weinberg, and Møller energy momentum complexes. In all the aforementioned prescriptions, the expressions for the energy of the gravitating system considered depend on the mass $M$ of the black hole, its charge $q$, a positive integer $\alpha$, and the radial coordinate $r$. In all these pseudotensorial prescriptions, the momenta are found to vanish, while the Landau-Lifshitz and Weinberg prescriptions give the same result for the energy distribution. In addition, the limiting behavior of the energy for the cases $r \rightarrow \infty, r \rightarrow 0$, and $q=0$ is studied. The special case $\mu=4$ and $\alpha=3$ is also examined. We conclude that the Einstein and Møller energy momentum complexes can be considered as the most reliable tools for the study of the energy momentum localization of a gravitating system.
\end{abstract}

\section{Introduction}

Energy momentum localization plays an important role among the open issues which appeared over the years in general relativity. The difficulty which arises consists in constructing a properly defined energy density of gravitating systems. As a result, up today there is no generally accepted satisfactory description for the energy of the gravitational field.

A number of researchers have used different methods for the energy momentum localization. Standard research methods include the use of different tools such as superenergy tensors [1-4], quasi-local expressions [5-9], and the well-known energy momentum complexes of Einstein [10-12], Landau-Lifshitz [13], Papapetrou [14], BergmannThomson [15], Møller [16], Weinberg [17], and Qadir-Sharif [18]. An agreement between, on one hand, Einstein, LandauLifshitz, Papapetrou, Bergmann-Thomson, Weinberg, and
Møller energy momentum complexes and, on the other hand, the quasi-local mass definition introduced by Penrose [19] and developed by Tod [20] for some gravitational backgrounds is worth noticing. The coordinate system dependence of these computational tools remains the main problem encountered. Indeed, only the Møller energy momentum complex is coordinate independent. "Cartesian coordinates" (also called quasi-Cartesian coordinates) and Kerr-Schild Cartesian coordinates are used to compute the energy momentum in the case of the other pseudotensorial prescriptions.

Despite the critique on energy momentum complexes, the 1990s have been proved to be a decade of rejuvenation of this field. This revival began in 1990 with the seminal research of Virbhadra [21, 22] who showed that the same energy distribution can be obtained for the (asymptotically Minkowskian) Kerr-Newman spacetime geometry by using different energy momentum prescriptions. In 1993, Rosen 
and Virbhadra [23] studied the energy momentum distribution of cylindrical gravitational waves by use of the Einstein prescription and it was shown, for the first time, that it was possible to obtain meaningful results also for asymptotically non-Minkowskian spacetimes. Furthermore, in 1996, it was shown by Aguirregabiria et al. [24] that several coordinate dependent prescriptions yield the same results for any metric of the Kerr-Schild class, while, in 1999, Virbhadra [25] extended the research for metrics more generally than the Kerr-Schild class obtaining also the same energy for different prescriptions. This line of research was enforced by Xulu [26] who by applying one more prescription found results in agreement with those of Virbhadra.

Since then, the aforementioned revival gave a boost to the field of energy momentum complexes with their application yielding physically reasonable results for many spacetime geometries, in particular for geometries in $(3+1),(2+1)$, and $(1+1)$ dimensions [27-48].

An alternative to avoid the dependence on coordinates is the teleparallel theory of gravitation [49-58] which has been used in many studies for the calculation of the energy and momentum distributions. Here, one can underline the similarity of the results obtained by this approach [59-63] with some of the results emerging by using the aforementioned standard energy momentum complexes.

One can also point out the ongoing attempts for the elaboration of the definition and application of energy momentum complexes as well as for their rehabilitation $[64,65]$.

Though the main motivation of knowing the nature of the spacetime itself by studying the energy momentum distributions remains, possible astrophysical implications also urge the research in this area. In his 1999 paper [25], Virbhadra studied the cosmic censorship hypothesis (CCH) and the Seifert conjecture by using several energy momentum complexes for investigating the "mass" of naked singularities. Building upon this work, gravitational lensing effects were attributed to naked singularities ([66] and references therein). A promising conjecture is that a positive energy region may act as a convergent gravitational lens, while a negative energy region may act as a divergent one (see, e.g., [67]). This line of research is still ongoing [68], whereas the determination of the effective gravitational mass of an object causing the curvature of spacetime, through energy momentum complexes, marks an independent line of research [69].

The present work has the following structure. In Section 2, we describe the new four-dimensional charged regular black hole solution under study. Section 3 contains the presentation of the Einstein, Landau-Lifshitz, Weinberg, and Møller energy momentum complexes utilized for the calculations. Section 4 contains the computations of the energy and momentum distributions. Finally, in the discussion given in Section 5, we present our results and examine some limiting and particular cases, while possible astrophysical perspectives are briefly mentioned. Throughout the paper we use geometrized units $(c=G=1)$, while the signature chosen is $(+,-,-,-)$. The calculations for the Einstein, LandauLifshitz, and Weinberg energy momentum complexes are performed in Schwarzschild Cartesian coordinates. Greek indices range from 0 to 3 , while Latin indices run from 1 to 3 .

\section{A New Regular Black Hole Solution in Einstein-Nonlinear Electrodynamics}

Attempts to avoid the central singularity in the presence of a horizon for a classical black hole have started already in the 1960s with Bardeen's regular black hole solution [70]. In fact, the interest in this area was revived after, by coupling gravity to certain theories of nonlinear electrodynamics, a number of exact solutions describing regular black holes (with all curvature invariants being bounded everywhere) were found (see [71] for a review of existing models and many relevant references). In this context, it is worth mentioning that the application of the Born-Infeld Lagrangian of nonlinear electrodynamics does not give a static spherically symmetric regular black hole solution since it creates conical singularities at the origin [72]. In 1998, Ayón-Beato and García [73] presented a regular black hole solution obtained by coupling gravity to nonlinear electrodynamics, whereby the Einstein-Hilbert action of general relativity is augmented by the addition to the Lagrangian of a term depending nonlinearly on the electromagnetic field tensor. Since then, a number of very interesting works have been published on this subject [74-84]. In fact, it is worth noticing that Ayón-Beato and García were the first to establish a connection of Bardeen's regular black hole solution with a nonlinear electrodynamics leading to the current interpretation of an exact solution supported by a nonlinear magnetic monopole [85].

The action for nonlinear electrodynamics minimally coupled to gravity used by Ayón-Beato and García is the following:

$$
S=\int d^{4} x \sqrt{-g}\left(\frac{1}{16 \pi} R-\frac{1}{4 \pi} \mathscr{L}(F)\right)
$$

where $R$ is the scalar curvature, while the gauge-invariant Lagrangian $\mathscr{L}(F)$ is a nonlinear function of the invariant $F=$ $(1 / 4) F_{\mu \nu} F^{\mu \nu}, F_{\mu \nu}$ being the electromagnetic field tensor. In the weak field limit, $\mathscr{L}(F)$ gives Maxwell's theory. Recently, Balart and Vagenas $[86,87]$ constructed a family of new spherical, static, and charged regular black hole solutions in the context of Einstein-nonlinear electrodynamics given above by imposing the following conditions: (i) the weak energy condition has to be satisfied, (ii) the energy momentum tensor has to respect the symmetry $T_{0}^{0}=T_{1}^{1}$ and, (iii) the metric must have an asymptotical behaviour as the Reissner-Nordström black hole solution. Starting with the general static and spherically symmetric line element,

$$
d s^{2}=B(r) d t^{2}-B^{-1}(r) d r^{2}-r^{2}\left(d \theta^{2}+\sin ^{2} \theta d \phi^{2}\right)
$$

with $B(r)=1-2 m(r) / r$ and, by relaxing the third condition, the authors have also constructed a more general family of charged regular black hole solutions which do not behave asymptotically as the Reissner-Nordström black hole metric. 
In this case, the mass function depends on two integer constant parameters $\mu \geqslant 4$ and $\alpha \geqslant 1$ :

$$
\begin{aligned}
& m(r)=\frac{r^{3} q^{2}}{6} \\
& \cdot \frac{\Gamma(1 / \alpha) \Gamma(\mu / \alpha)}{\Gamma(4 / \alpha) \Gamma((\mu-3) / \alpha)}\left(\frac{6 \Gamma(4 / \alpha)}{\Gamma(1 / \alpha) \Gamma((\alpha+3) / \alpha)} \frac{M}{q^{2}}\right)^{4} \\
& \quad \cdot{ }_{2} F_{1}\left(\frac{3}{\alpha}, \frac{\mu}{\alpha}, \frac{3+\alpha}{\alpha} ;\right. \\
& \left.-\left(\frac{6 \Gamma(4 / \alpha)}{\Gamma(1 / \alpha) \Gamma((\alpha+3) / \alpha)} \frac{M}{q^{2}} r\right)^{\alpha}\right),
\end{aligned}
$$

where $q$ is the electric charge, $\Gamma$ is the Gamma function, $M$ is the black hole mass, and ${ }_{2} F_{1}$ is the Gauss hypergeometric function. Particular cases such as $\mu=5$ and $\alpha=2$ and $\mu=6$ and $\alpha=3$ lead to the metrics obtained by Bardeen [70] and Hayward [88].

The line element (2) with the mass function (3) does not behave asymptotically as the Reissner-Nordström black hole solution unless $\mu=4$. In fact, for $\mu=4$ and $\alpha=3$ in (3), Balart and Vagenas obtained the asymptotically ReissnerNordström solution

$$
B(r)=1-\frac{2 M}{r}\left[1-\frac{1}{\left[1+\left(2 M r / q^{2}\right)^{3}\right]^{1 / 3}}\right] .
$$

In the present paper, we will study the nonasymptotically Reissner-Nordström gravitational background obtained for the particular case $\mu=3$ and $\alpha$ arbitrary. Consequently, the metric for the new charged regular black hole solution to be considered in what follows has the line element (2) with

$$
B(r)=1-\frac{2 M}{r}\left(1-\frac{1}{\left[1+\left(2 M r / q^{2}\right)^{3}\right]^{(\alpha-3) / 3}}\right) .
$$

In fact, this regular black hole solution exhibits two event horizons. By choosing, for example, the numerical values $M=1$ and $q=0.9$, we get Figure 1 showing the two horizons. One can see that the position of the inner horizon is shifted towards bigger values of $r$ as the parameter $\alpha$ decreases, while the position of the outer horizon remains unaffected.

\section{Einstein, Landau-Lifshitz, Weinberg, and Møller Energy Momentum Complexes}

The Einstein energy momentum complex $[10,11]$ for a $(3+$ 1) dimensional gravitational background is given by the expression

$$
\theta_{\nu}^{\mu}=\frac{1}{16 \pi} h_{v, \lambda}^{\mu \lambda}
$$

where the superpotentials $h_{v}^{\mu \lambda}$ are given as

$$
h_{\nu}^{\mu \lambda}=\frac{1}{\sqrt{-g}} g_{\nu \sigma}\left[-g\left(g^{\mu \sigma} g^{\lambda \kappa}-g^{\lambda \sigma} g^{\mu \kappa}\right)\right]_{, \kappa}
$$

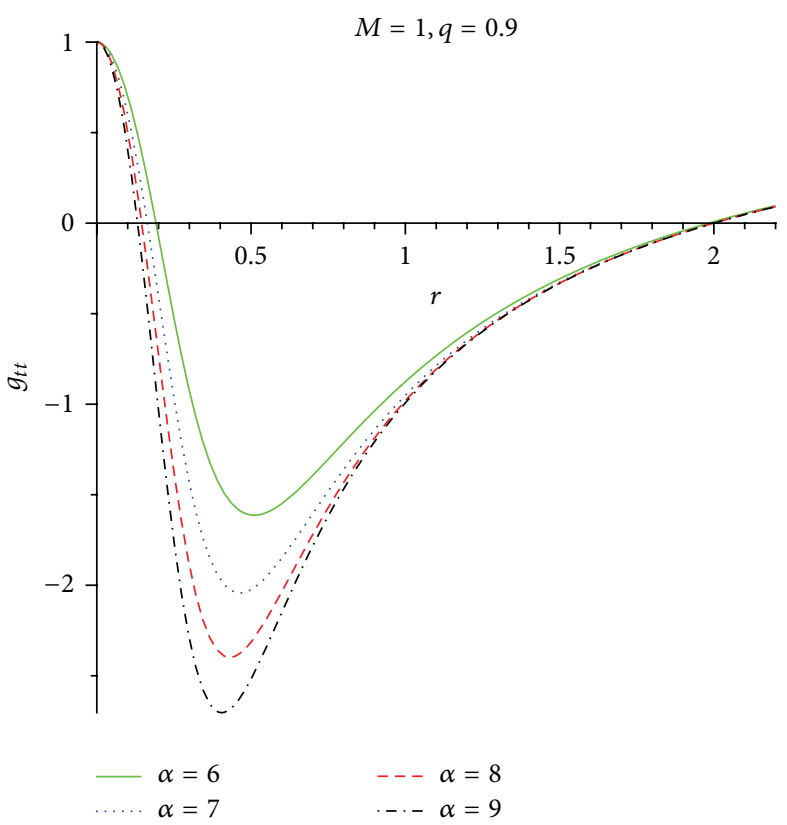

FIgURE 1: Two horizons exist for the charged regular black hole at the points where $g_{t t}$ meets the $r$-axis. For different values of the parameter $\alpha$, the positions of the inner horizon assume different values, whereas the position of the outer horizon remains unaffected.

and satisfy the corresponding antisymmetric property

$$
h_{v}^{\mu \lambda}=-h_{v}^{\lambda \mu}
$$

The components $\theta_{0}^{0}$ and $\theta_{i}^{0}$ represent the energy and the momentum densities, respectively. In this prescription, the local conservation law is respected:

$$
\theta_{\nu, \mu}^{\mu}=0 .
$$

Thus, the energy momentum can be calculated by

$$
P_{\mu}=\iiint \theta_{\mu}^{0} d x^{1} d x^{2} d x^{3} .
$$

Applying Gauss's theorem, the energy momentum becomes

$$
P_{\mu}=\frac{1}{16 \pi} \iint h_{\mu}^{0 i} n_{i} d S
$$

with $n_{i}$ being the outward unit normal vector on the surface $d S$.

In the Landau-Lifshitz prescription, the corresponding energy momentum complex [13] is defined as

$$
L^{\mu \nu}=\frac{1}{16 \pi} S_{, \rho \sigma}^{\mu \nu \rho \sigma},
$$

with the Landau-Lifshitz superpotentials given by

$$
S^{\mu \nu \rho \sigma}=-g\left(g^{\mu \nu} g^{\rho \sigma}-g^{\mu \rho} g^{\nu \sigma}\right) .
$$

$L^{00}$ and $L^{0 i}$ components are the energy and the momentum densities, respectively. The local conservation law holds:

$$
L_{, v}^{\mu \nu}=0 .
$$


By integrating $L^{\mu \nu}$ over the 3-space one gets for the energy momentum

$$
P^{\mu}=\iiint L^{\mu 0} d x^{1} d x^{2} d x^{3}
$$

By using Gauss's theorem, we get

$$
P^{\mu}=\frac{1}{16 \pi} \iint S_{, v}^{\mu 0 i v} n_{i} d S=\frac{1}{16 \pi} \iint U^{\mu 0 i} n_{i} d S
$$
as

The Weinberg energy momentum complex [17] is given

$$
W^{\mu \nu}=\frac{1}{16 \pi} D_{, \lambda}^{\lambda \mu \nu}
$$

where $D^{\lambda \mu \nu}$ represents the corresponding superpotentials:

$$
\begin{aligned}
D^{\lambda \mu \nu}= & \frac{\partial h_{\kappa}^{\kappa}}{\partial x_{\lambda}} \eta^{\mu \nu}-\frac{\partial h_{\kappa}^{\kappa}}{\partial x_{\mu}} \eta^{\lambda \nu}-\frac{\partial h^{\kappa \lambda}}{\partial x^{\kappa}} \eta^{\mu \nu}+\frac{\partial h^{\kappa \mu}}{\partial x^{\kappa}} \eta^{\lambda \nu} \\
& +\frac{\partial h^{\lambda \nu}}{\partial x_{\mu}}-\frac{\partial h^{\mu \nu}}{\partial x_{\lambda}},
\end{aligned}
$$

with

$$
h_{\mu \nu}=g_{\mu \nu}-\eta_{\mu \nu}
$$

Here $W^{00}$ and $W^{0 i}$ components represent the energy and the momentum densities, respectively. In the Weinberg prescription, the local conservation law is also respected:

$$
W_{, \nu}^{\mu \nu}=0
$$

The integration of $W^{\mu \nu}$ over the 3-space gives the energy momentum

$$
P^{\mu}=\iiint W^{\mu 0} d x^{1} d x^{2} d x^{3}
$$

Applying Gauss's theorem and integrating over the surface of a sphere of radius $r$, we get the following expression for the energy momentum distribution:

$$
P^{\mu}=\frac{1}{16 \pi} \iint D^{i 0 \mu} n_{i} d S
$$

The Møller energy momentum complex [16] is

$$
\mathscr{J}_{v}^{\mu}=\frac{1}{8 \pi} M_{\nu, \lambda}^{\mu \lambda}
$$

with the Møller superpotentials $M_{v}^{\mu \lambda}$,

$$
M_{\nu}^{\mu \lambda}=\sqrt{-g}\left(\frac{\partial g_{\nu \sigma}}{\partial x^{\kappa}}-\frac{\partial g_{\nu \kappa}}{\partial x^{\sigma}}\right) g^{\mu \kappa} g^{\lambda \sigma}
$$

being antisymmetric

$$
M_{v}^{\mu \lambda}=-M_{v}^{\lambda \mu}
$$

Møller's energy momentum complex also satisfies the local conservation law:

$$
\frac{\partial \mathscr{F}_{v}^{\mu}}{\partial x^{\mu}}=0
$$

while $\mathscr{F}_{0}^{0}$ and $\mathscr{F}_{i}^{0}$ are the energy and the momentum densities, respectively. Thus, the energy momentum is given by

$$
P_{\mu}=\iiint \mathscr{J}_{\mu}^{0} d x^{1} d x^{2} d x^{3}
$$

With the use of Gauss's theorem, one gets

$$
P_{\mu}=\frac{1}{8 \pi} \iint M_{\mu}^{0 i} n_{i} d S
$$

\section{Energy and Momentum Distributions for the Charged Regular Black Hole}

In order to perform the calculations using the Einstein energy momentum complex, it is necessary to transform the metric given by the line element (2) in Schwarzschild Cartesian coordinates. Thus, we obtain the line element in the following form:

$$
\begin{aligned}
d s^{2}= & B(r) d t^{2}-\left(d x^{2}+d y^{2}+d z^{2}\right) \\
& -\frac{B^{-1}(r)-1}{r^{2}}(x d x+y d y+z d z)^{2} .
\end{aligned}
$$

Now, after computing the superpotentials in the Einstein prescription, we get the following nonvanishing components:

$$
\begin{aligned}
& h_{0}^{01}=\frac{2 x}{r^{2}} \frac{2 M}{r}\left\{1-\frac{1}{\left[1+\left(2 M r / q^{2}\right)^{3}\right]^{(\alpha-3) / 3}}\right\}, \\
& h_{0}^{02}=\frac{2 y}{r^{2}} \frac{2 M}{r}\left\{1-\frac{1}{\left[1+\left(2 M r / q^{2}\right)^{3}\right]^{(\alpha-3) / 3}}\right\}, \\
& h_{0}^{03}=\frac{2 z}{r^{2}} \frac{2 M}{r}\left\{1-\frac{1}{\left[1+\left(2 M r / q^{2}\right)^{3}\right]^{(\alpha-3) / 3}}\right\} .
\end{aligned}
$$

Using the line element (2), with the metric coefficient (5), (11) for the energy and the expressions, and (30) for the superpotentials, we obtain the energy distribution for the nonasymptotically Reissner-Nordström regular charged black hole in the Einstein prescription:

$$
E_{E}=M\left\{1-\frac{1}{\left[1+\left(2 M r / q^{2}\right)^{3}\right]^{(\alpha-3) / 3}}\right\} \text {. }
$$

Furthermore, all the momenta are zero. 


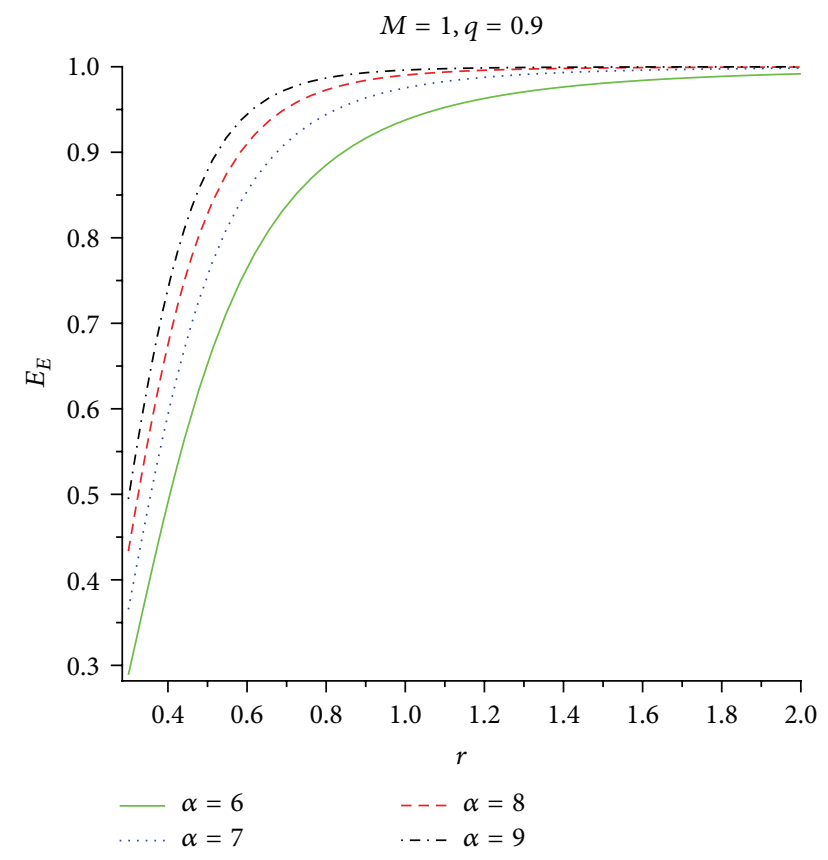

FIGURE 2: Energy distribution calculated by the Einstein prescription between the inner and outer horizons for different values of the parameter $\alpha$.

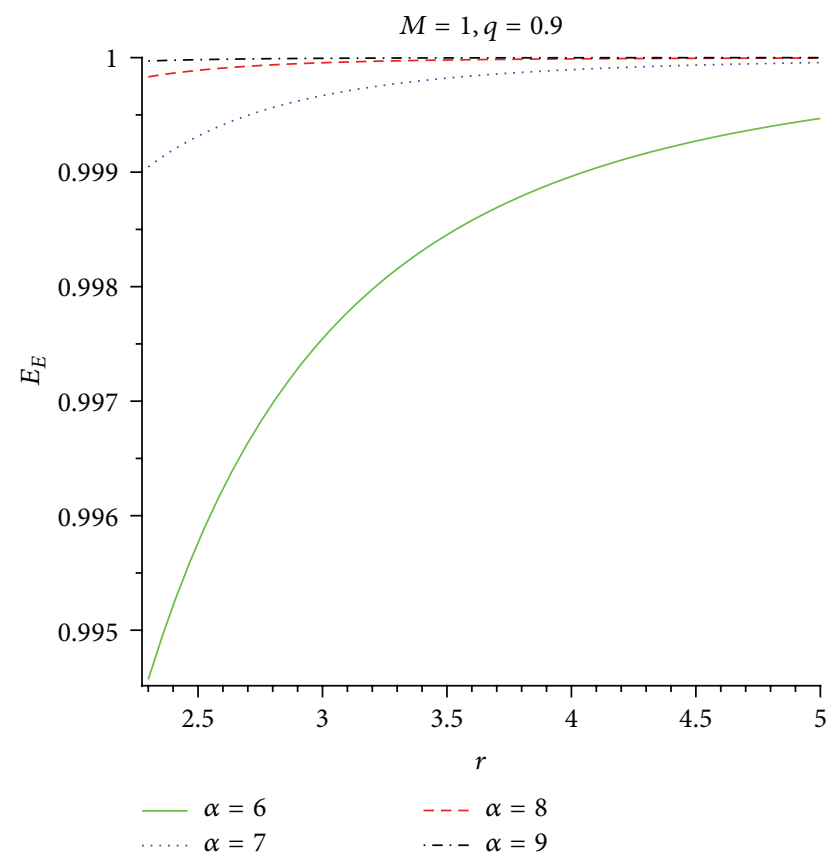

FIgURE 3: Energy distribution calculated by the Einstein prescription outside the outer horizon for different values of the parameter $\alpha$.

In Figures 2 and 3, we can see the graphs of the energy distribution as a function of the distance for different values of $\alpha$ between the two horizons and outside the outer horizon, respectively, while Figure 4 presents the energy distribution as a function of charge and mass.

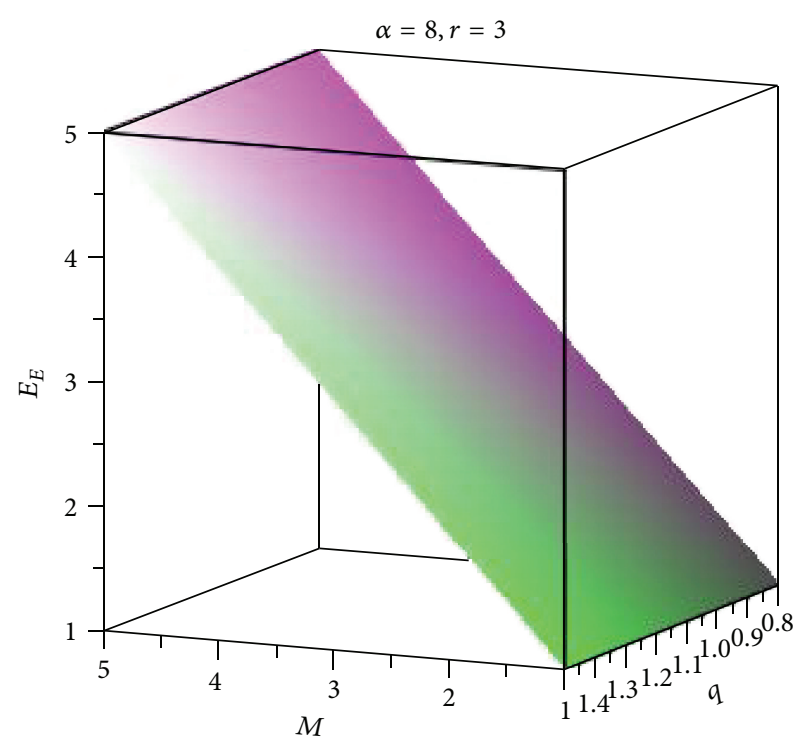

FIGURE 4: Variation of the energy distribution calculated by the Einstein prescription with respect to charge and mass outside the outer horizon.

In order to apply the Landau-Lifshitz prescription, we use $U^{\mu 0 i}$ quantities defined in (16) to compute the energy momentum distribution. The nonvanishing components of $U^{\mu 0 i}$ quantities are

$$
\begin{aligned}
& U^{t t x} \\
& =\frac{2 x}{r^{2}} \frac{(2 M / r)\left[1-1 /\left[1+\left(2 M r / q^{2}\right)^{3}\right]^{(\alpha-3) / 3}\right]}{1-(2 M / r)\left[1-1 /\left[1+\left(2 M r / q^{2}\right)^{3}\right]^{(\alpha-3) / 3}\right]}, \\
& U^{t t y} \\
& =\frac{2 y}{r^{2}} \frac{(2 M / r)\left[1-1 /\left[1+\left(2 M r / q^{2}\right)^{3}\right]^{(\alpha-3) / 3}\right]}{1-(2 M / r)\left[1-1 /\left[1+\left(2 M r / q^{2}\right)^{3}\right]^{(\alpha-3) / 3}\right]}, \\
& U^{t t z}, \\
& =\frac{2 z}{r^{2}} \frac{(2 M / r)\left[1-1 /\left(1+\left(2 M r / q^{2}\right)^{3}\right)^{(\alpha-3) / 3}\right]}{1-(2 M / r)\left[1-1 /\left[1+\left(2 M r / q^{2}\right)^{3}\right]^{(\alpha-3) / 3}\right]} .
\end{aligned}
$$

Substituting (32) in (16), we get the energy distribution

$$
E_{L L}=\frac{M\left[1-1 /\left[1+\left(2 M r / q^{2}\right)^{3}\right]^{(\alpha-3) / 3}\right]}{1-(2 M / r)\left[1-1 /\left[1+\left(2 M r / q^{2}\right)^{3}\right]^{(\alpha-3) / 3}\right]} .
$$

Also, for this prescription, all the momenta are found to vanish. 
In the case of the Weinberg prescription, we calculate the nonvanishing components:

$$
\begin{aligned}
& D^{x t t} \\
& =\frac{2 x}{r^{2}} \frac{(2 M / r)\left[1-1 /\left[1+\left(2 M r / q^{2}\right)^{3}\right]^{(\alpha-3) / 3}\right]}{1-(2 M / r)\left[1-1 /\left[1+\left(2 M r / q^{2}\right)^{3}\right]^{(\alpha-3) / 3}\right]}, \\
& D^{y t t} \\
& =\frac{2 y}{r^{2}} \frac{(2 M / r)\left[1-1 /\left[1+\left(2 M r / q^{2}\right)^{3}\right]^{(\alpha-3) / 3}\right]}{1-(2 M / r)\left[1-1 /\left[1+\left(2 M r / q^{2}\right)^{3}\right]^{(\alpha-3) / 3}\right]}, \\
& \left.D^{z t t}\right] \\
& =\frac{2 z}{r^{2}} \frac{(2 M / r)\left[1-1 /\left[1+\left(2 M r / q^{2}\right)^{3}\right]^{(\alpha-3) / 3}\right]}{1-(2 M / r)\left[1-1 /\left[1+\left(2 M r / q^{2}\right)^{3}\right]^{(\alpha-3) / 3}\right]} .
\end{aligned}
$$

Inserting the expressions obtained in (34) into (22), we obtain for the energy distribution inside a 2 -sphere of radius $r$

$$
\begin{aligned}
& E_{W} \\
& =M \frac{\left[1-1 /\left[1+\left(2 M r / q^{2}\right)^{3}\right]^{(\alpha-3) / 3}\right]}{1-(2 M / r)\left[1-1 /\left[1+\left(2 M r / q^{2}\right)^{3}\right]^{(\alpha-3) / 3}\right]},
\end{aligned}
$$

while all the momenta vanish. As it can be seen, the energy in the Weinberg prescription (35) is identical with the energy in the Landau-Lifshitz prescription (33).

In Figures 5 and 6, we can see the graphs of the energy distribution as a function of the distance for different values of $\alpha$ between the two horizons and outside the outer horizon, respectively, while Figure 7 presents the energy distribution as a function of charge and mass.

In the Møller prescription, the only nonvanishing superpotential is

$$
\begin{gathered}
M_{0}^{01}=\left\{\frac{2 M\left[1-1 /\left(1+8 M^{3} r^{3} / q^{6}\right)^{(\alpha / 3-1)}\right]}{r^{2}}\right. \\
\left.-\frac{48 M^{4}(\alpha / 3-1) r}{\left[1+8 M^{3} r^{3} / q^{6}\right]^{(\alpha / 3-1)} q^{6}\left[1+8 M^{3} r^{3} / q^{6}\right]}\right\} r^{2}
\end{gathered}
$$

$\cdot \sin \theta$.

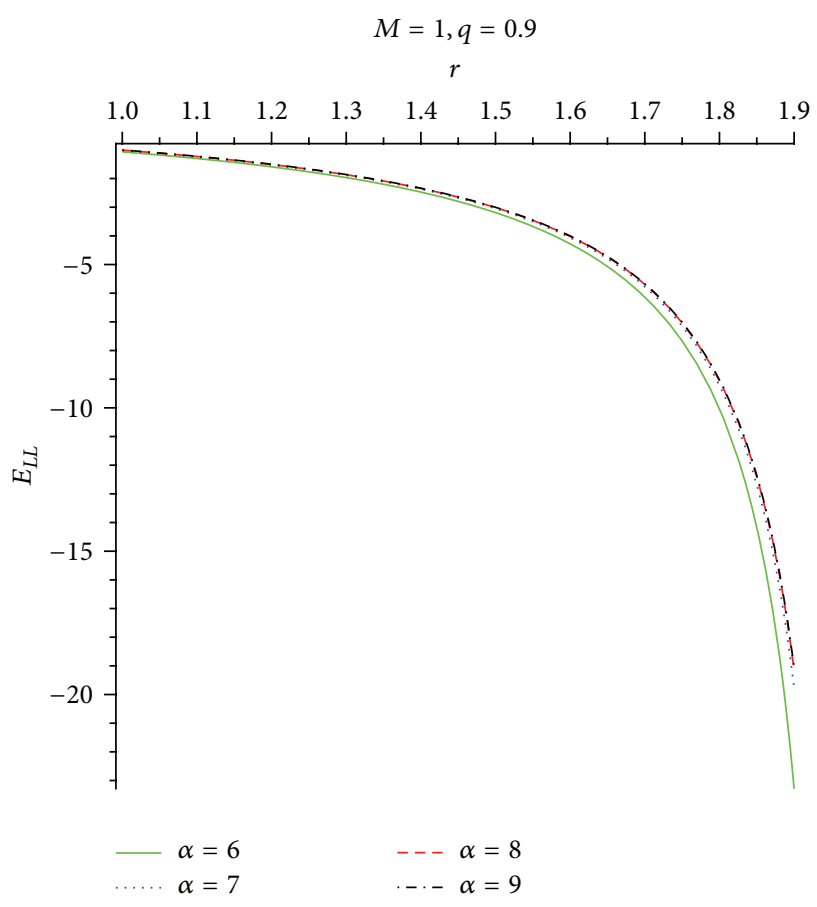

FIGURE 5: Energy distribution calculated by the Landau-Lifshitz prescription between the inner and outer horizons for different values of the parameter $\alpha$.

Applying the last result for the line element (2) with the metric coefficient (5) and using the expression (28), we get the energy distribution in the Møller prescription:

$$
\begin{aligned}
E_{M} & =\frac{r^{2}}{2}\left\{\frac{2 M\left[1-1 /\left(1+8 M^{3} r^{3} / q^{6}\right)^{(\alpha / 3-1)}\right]}{r^{2}}\right. \\
& \left.-\frac{48 M^{4}(\alpha / 3-1) r}{\left[1+8 M^{3} r^{3} / q^{6}\right]^{(\alpha / 3-1)} q^{6}\left[1+8 M^{3} r^{3} / q^{6}\right]}\right\} .
\end{aligned}
$$

Also, in this prescription, it is found that all the momenta vanish.

In Figures 8 and 9, we can see the graphs of the energy distribution as a function of the distance for different values of $\alpha$ between the two horizons and outside the outer horizon, respectively, while Figure 10 presents the energy distribution as a function of charge and mass.

It is pointed out, according to Figure 8 , that for $\alpha \leqslant 6$ the Møller energy of the regular black hole is negative and decreases monotonically for the whole range of the values of $r$ considered.

Finally, in Figure 11, we give a comparison of the energy distributions as obtained by the different prescriptions for $\alpha=$ 8. 


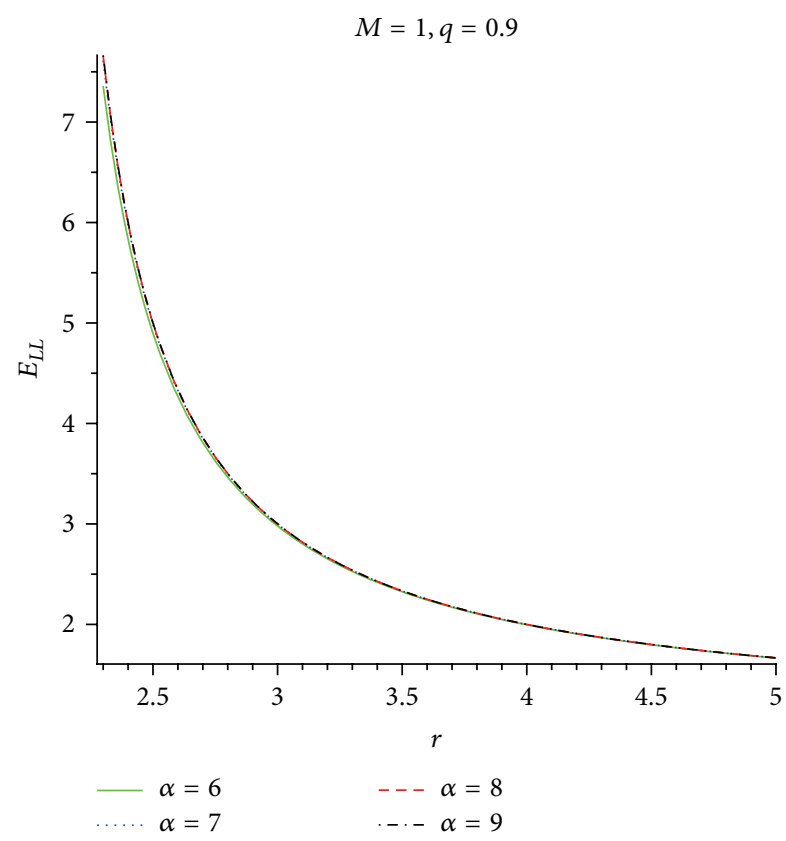

Figure 6: Energy distribution calculated by the Landau-Lifshitz prescription outside the outer horizon for different values of the parameter $\alpha$.

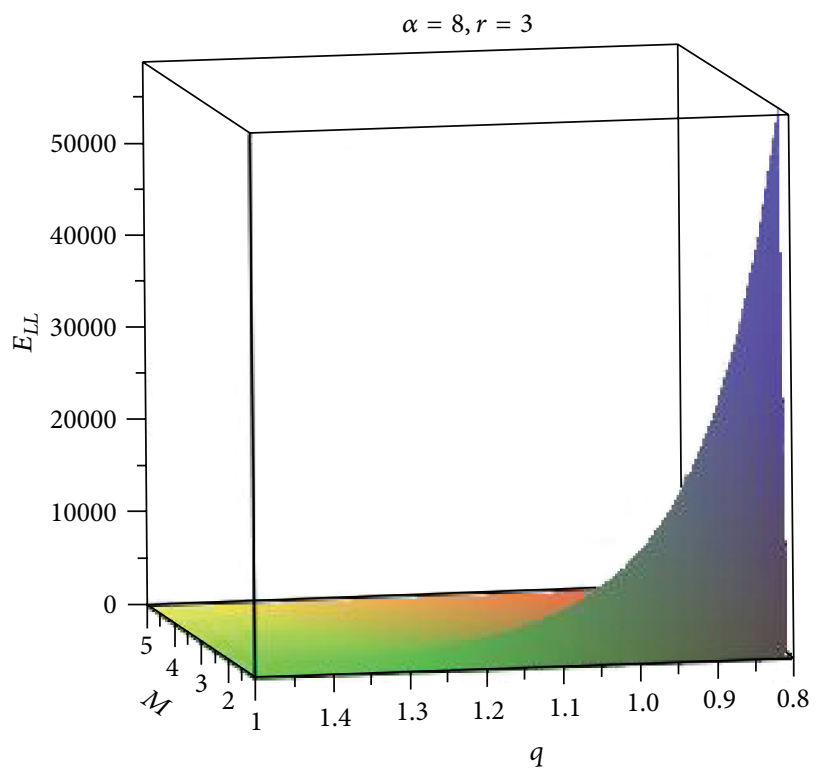

FIgURE 7: Variation of the energy distribution calculated by the Landau-Lifshitz prescription with respect to charge and mass outside the outer horizon.

\section{Discussion}

This paper is focused on the study of the energy momentum for the gravitational field of a new four-dimensional, spherically symmetric, static and charged, and regular black hole solution developed in the context of a particular Einstein-nonlinear electrodynamics coupling, by applying the Einstein, Landau-Lifshitz, Weinberg, and Møller energy

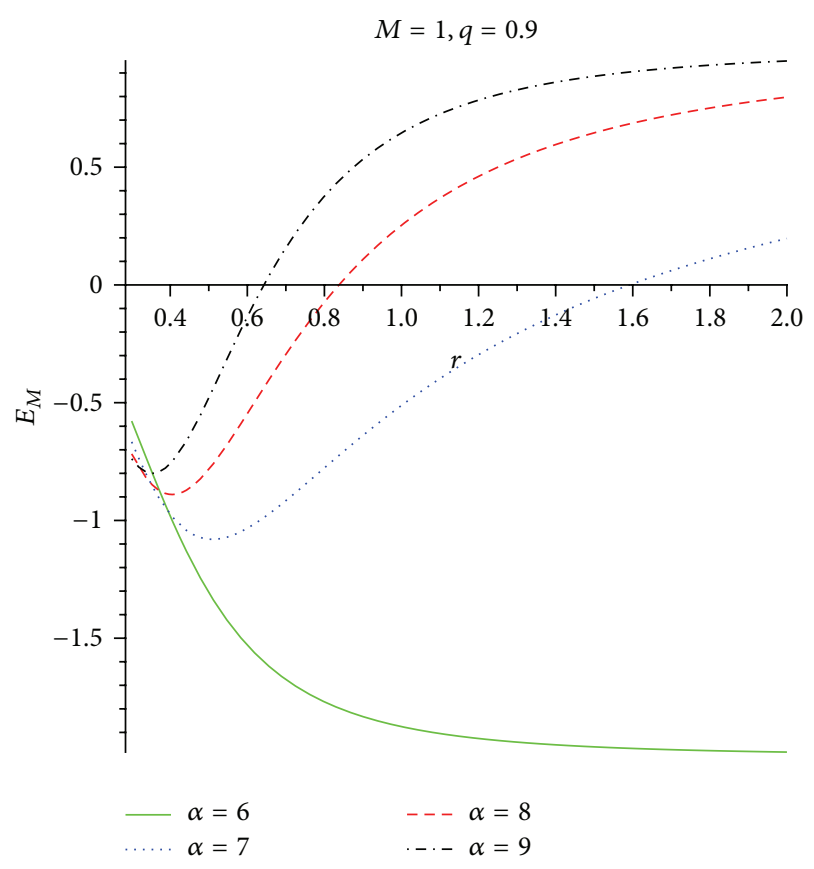

FIGURE 8: Energy distribution calculated by the Møller prescription between the inner and outer horizons for different values of the parameter $\alpha$.

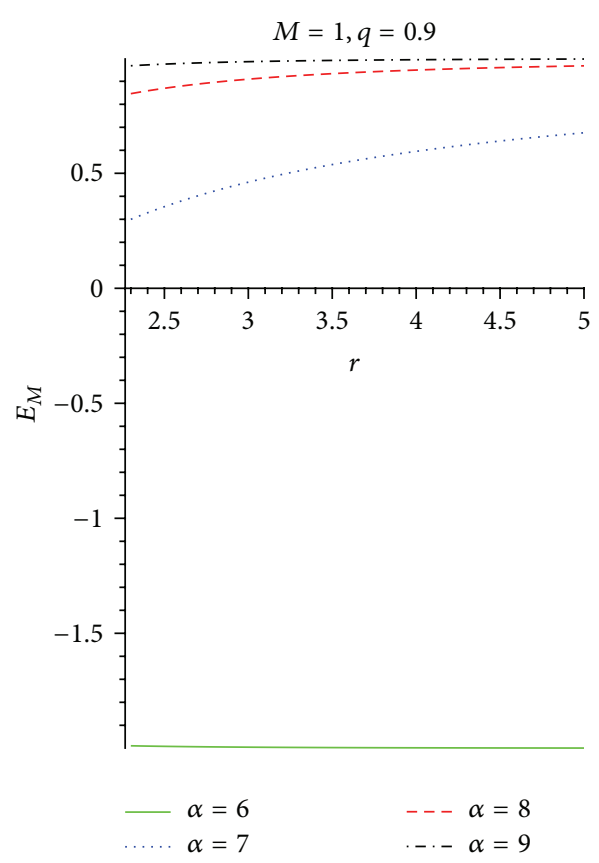

FIGURE 9: Energy distribution calculated by the Møller prescription outside the outer horizon for different values of the parameter $\alpha$.

momentum complexes. The metric considered, which has two horizons, does not asymptotically behave as the ReissnerNordström black hole solution unless $\mu=4$, where $\mu$ is a positive integer parameter. The expressions for the energy are well defined in all the aforementioned prescriptions and depend on the mass $M$ of the black hole, its charge $q$, a 


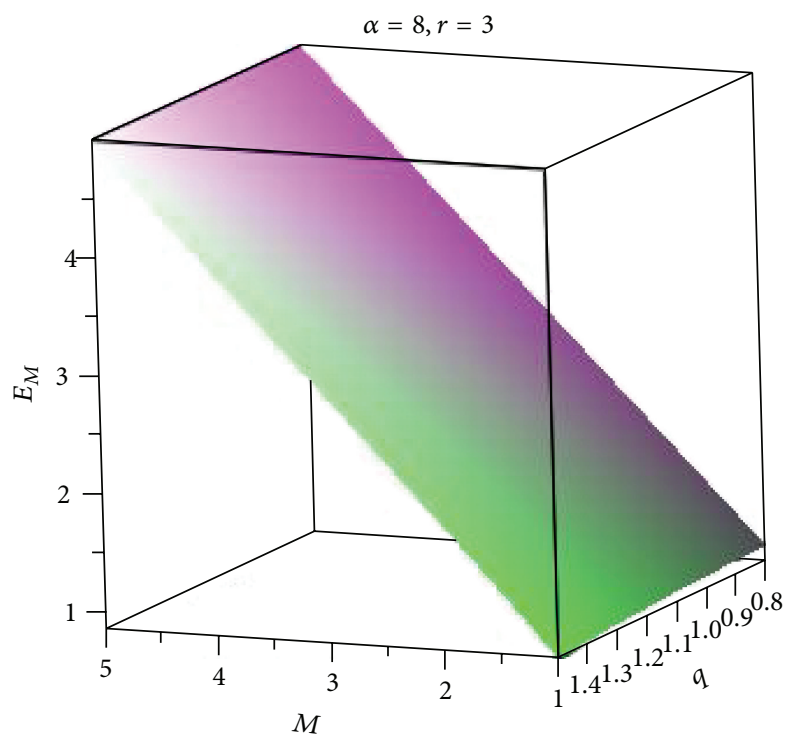

FIGURE 10: Variation of the energy distribution calculated by the Møller prescription with respect to charge and mass outside the outer horizon.

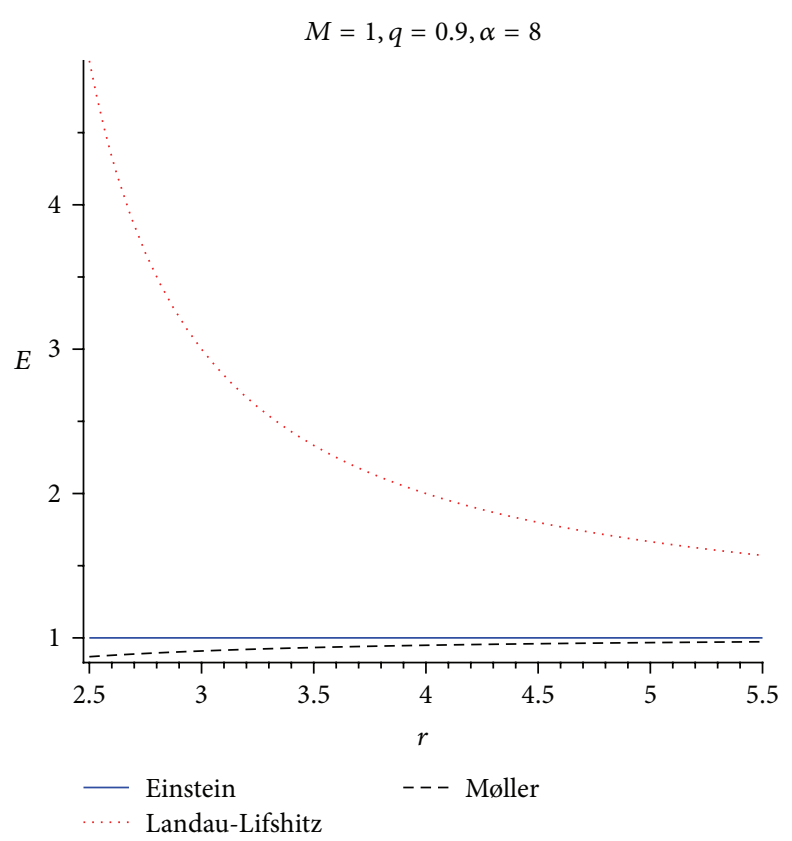

FIGURE 11: Comparison of the energy distributions calculated by the Einstein, Landau-Lifshitz, and Møller prescriptions outside the outer horizon.

positive integer $\alpha$, and the radial coordinate $r$. Both the Landau-Lifshitz and Weinberg prescriptions give the same result for the energy distribution, while all the momenta are zero in all four prescriptions used. In Table 1, we present the results for the energy as obtained in the four different prescriptions for the nonasymptotically Reissner-Nordström regular charged black hole.

In order to examine the physical meaning of the results obtained, we study the limiting behavior of the energy for $r \rightarrow \infty, r \rightarrow 0$, and $q=0$ in three cases: (i) $1 \leqslant \alpha<3$, (ii) $\alpha=3$, and (iii) $\alpha>3$. However, since cases (i) and (ii) do not yield any physically meaningful results, we present only the results for case (iii) in Table 2.

For $\mu=4$ and $\alpha=3$, we calculate the energy momentum distribution for the asymptotically ReissnerNordström metric with the metric coefficient (4). For this black hole solution, the energy distribution obtained by using the Einstein, Landau-Lifshitz, Weinberg, and Møller energy 
TABLE 1: Energy calculated for the nonasymptotically Reissner-Nordström regular charged black hole ( $\mu=3, \alpha$ arbitrary) in the different prescriptions.

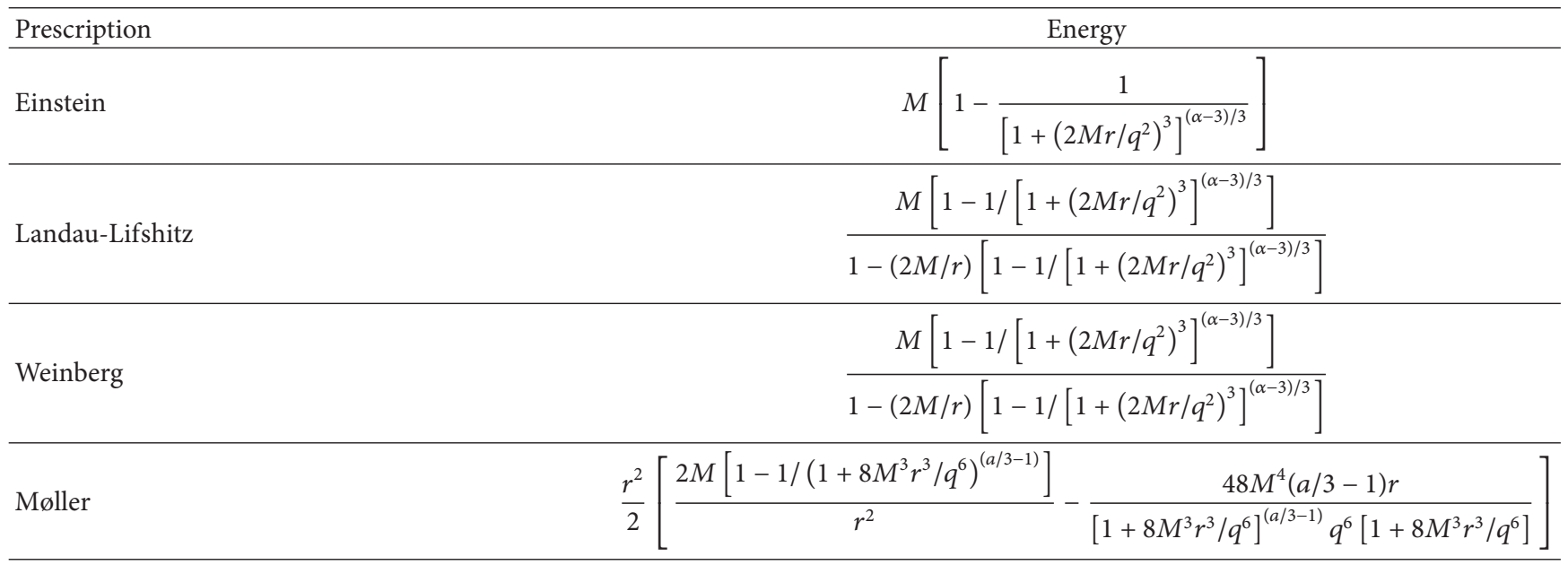

TABLE 2: Energy for $\alpha>3$ in the different prescriptions at limiting cases.

\begin{tabular}{lccc}
\hline Prescription & $r \rightarrow \infty$ & $r \rightarrow 0$ & $q=0$ \\
\hline Einstein & $M$ & 0 & $M$ \\
Landau-Lifshitz & $M$ & 0 & $M\left(1-\frac{2 M}{r}\right)^{-1}$ \\
Weinberg & $M$ & 0 & $M\left(1-\frac{2 M}{r}\right)^{-1}$ \\
Møller & $M$ & 0 & $M$ \\
\hline
\end{tabular}

momentum complexes is given in Table 3, along with the limiting cases for $r \rightarrow \infty, r \rightarrow 0$, and $q=0$. As we can see, the limiting behavior of the energy for the asymptotically Reissner-Nordström solution ( $\mu=4, \alpha=3$ ) is the same as in the case of the nonasymptotically Reissner-Nordström solution given in Table 2 .

Thus, by comparing the results obtained for the energy with all the energy momentum complexes applied, we see that, for $q=0$ and at infinity, the Einstein prescription and the Møller prescription give the same results (namely, the ADM mass $M$ ) as those obtained for the Schwarzschild black hole solution. On the other hand, the Landau-Lifshitz and the Weinberg prescriptions, while at infinity lead to the same result (the ADM mass $M$ ), in the chargeless case $q=0$ give the energy distribution $E_{L L}=E_{W}=M(1-2 M / r)^{-1}$ which is in total agreement with the expression obtained for the Schwarzschild black hole using Schwarzschild Cartesian coordinates (see, [25]).

Finally, all the four prescriptions used yield a vanishing energy as $r \rightarrow 0$.

Based on the present study, as well as on the aforementioned agreement with previous results obtained in the case of the Schwarzschild metric, we conclude that the Einstein and Møller energy momentum complexes give physically meaningful results and, thus, can be considered as the most reliable tools among the energy momentum complexes for the study of the energy momentum localization of gravitational systems.

At this point, we would like to mention a possible astrophysical implication of our results. Identifying [89] the energy distribution at radial distance $r$ with the effective gravitational mass of the astrophysical object considered inside the region determined by this distance $r$, it would be possible to investigate whether this effective gravitational mass is positive or negative and decide accordingly if the astrophysical object could act as a convergent or divergent gravitational lens. This investigation, though, lies beyond the scope of the present work.

Another interesting future perspective might be the calculation of the energy momentum of the new regular black hole solution considered in the present work by applying other energy momentum complexes and/or the teleparallel equivalent to general relativity (TEGR).

A final remark is deemed proper. Recently, it has been shown that the Maxwell-modified $F(R)$ theory of gravity with an action of the form $S=\int d^{4} x \sqrt{-g}\left[F(R)-(1 / 4) F_{\mu \nu} F^{\mu \nu}\right]$ allows the occurrence of instability and antievaporation effects for charged (in particular, extremal ReissnerNordström) black holes [90]. Since Einstein-nonlinear electrodynamics can also be considered as a kind of modified gravity theory, similar instability and antievaporation effects can in principle be expected to occur. Their study will shed new light on the behavior of classical black holes.

\section{Conflict of Interests}

The authors declare that there is no conflict of interests regarding the publication of this paper. 
TABLE 3: Energy of the asymptotically Reissner-Nordström regular solution $(\mu=4$ and $\alpha=3)$ in the different prescriptions as well as in limiting cases and in the uncharged case.

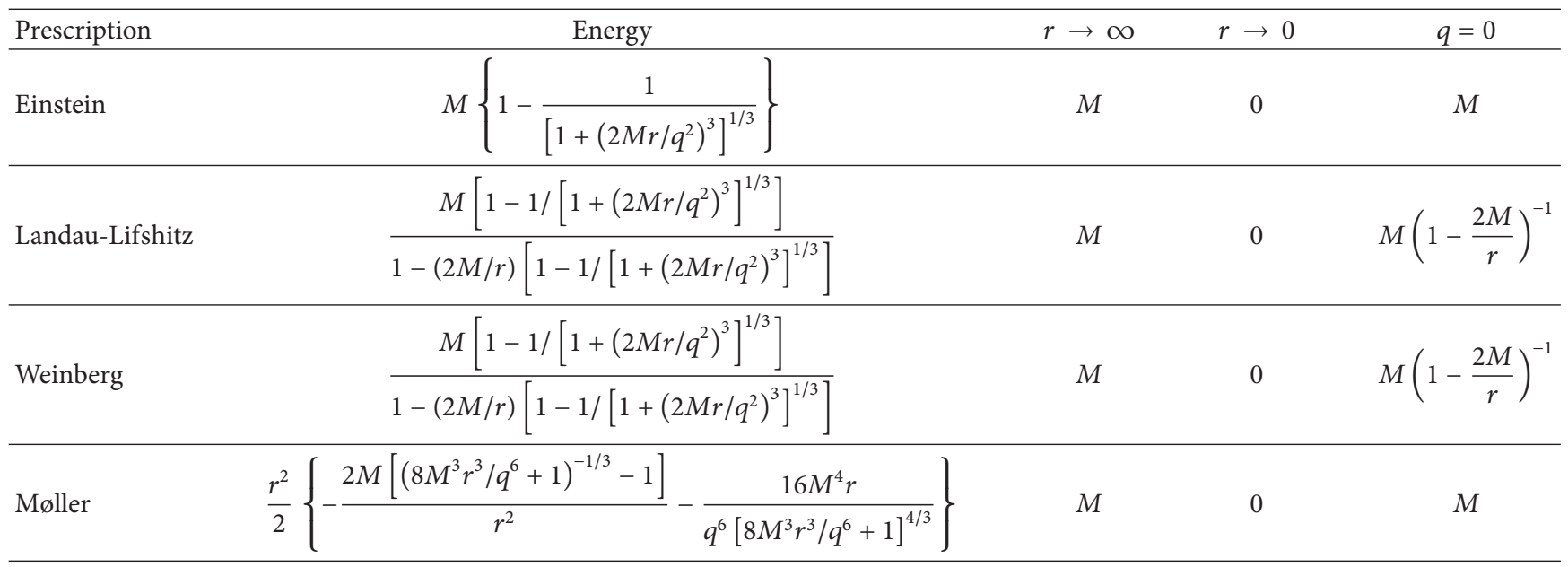

\section{Acknowledgments}

The authors are thankful to the anonymous referees for their helpful comments and suggestions. Sayeedul Islam is also thankful to DST, Government of India, for providing financial support under INSPIRE Fellowship.

\section{References}

[1] L. Bel, "Définition d'une densité d'énergie et d'un état de radiation totale généralisée," Comptes Rendus Hebdomadaires des Séances de l’Académie des Sciences, vol. 246, pp. 3015-3018, 1958.

[2] I. Robinson, "On the Bel-Robinson tensor," Classical and Quantum Gravity, vol. 14, no. 1A, pp. A331-A333, 1997.

[3] M. A. G. Bonilla and J. M. M. Senovilla, "Some properties of the BEL and BEL-Robinson tensors," General Relativity and Gravitation, vol. 29, no. 1, pp. 91-116, 1997.

[4] J. M. Senovilla, "Super-energy tensors," Classical and Quantum Gravity, vol. 17, no. 14, pp. 2799-2841, 2000.

[5] J. D. Brown and J. W. York Jr., "Quasilocal energy and conserved charges derived from the gravitational action," Physical Review D, vol. 47, no. 4, pp. 1407-1419, 1993.

[6] S. A. Hayward, "Quasilocal gravitational energy," Physical Review D, vol. 49, no. 2, pp. 831-839, 1994.

[7] C.-M. Chen and J. M. Nester, "Quasilocal quantities for general relativity and other gravity theories," Classical and Quantum Gravity, vol. 16, no. 4, pp. 1279-1304, 1999.

[8] C.-C. M. Liu and S.-T. Yau, "Positivity of quasilocal mass," Physical Review Letters, vol. 90, no. 23, Article ID 231102, 2003.

[9] L. Balart, "Quasilocal energy, Komar charge and horizon for regular black holes," Physics Letters B, vol. 687, no. 4-5, pp. 280$285,2010$.

[10] A. Einstein, "Zur allgemeinen Relativitätstheorie," Sitzungsberichte der Königlich Preussischen Akademie der Wissenschaften zu Berlin, vol. 47, pp. 778-786, 1915.
[11] A. Einstein, "Zur allgemeinen Relativitätstheorie (Nachtrag)," Sitzungsberichte der Königlich Preussischen Akademie der Wissenschaften zu Berlin, vol. 47, pp. 799-801, 1915.

[12] A. Trautman, "Conservation laws in general relativity," in Gravitation: An Introduction to Current Research, L. Witten, Ed., pp. 169-198, John Wiley \& Sons, New York, NY, USA, 1962.

[13] L. D. Landau and E. M. Lifshitz, The Classical Theory of Fields, Pergamon Press, 1987.

[14] A. Papapetrou, "Einstein's theory of gravitation and flat space," Proceedings of the Royal Irish Academy A, vol. 52, pp. 11-23, 1948.

[15] P. G. Bergmann and R. Thomson, "Spin and angular momentum in general relativity," Physical Review, vol. 89, no. 2, pp. 400-407, 1953.

[16] C. Møller, "On the localization of the energy of a physical system in the general theory of relativity," Annals of Physics, vol. 4, no. 4, pp. 347-371, 1958.

[17] S. Weinberg, Gravitation and Cosmology: Principles and Applications of General Theory of Relativity, John Wiley \& Sons, New York, NY, USA, 1972.

[18] A. Qadir and M. Sharif, "General formula for the momentum imparted to test particles in arbitrary spacetimes," Physics Letters A, vol. 167, no. 4, pp. 331-334, 1992.

[19] R. Penrose, "Quasilocal mass and angular momentum in general relativity," Proceedings of the Royal Society of London, Series A: Mathematical and Physical Sciences, vol. 381, no. 1780, pp. 5363, 1982.

[20] K. P. Tod, "Some examples of Penrose's quasi-local mass construction," Proceedings of the Royal Society of London A, vol. 388, no. 1795, pp. 457-477, 1983.

[21] K. S. Virbhadra, "Energy associated with a Kerr-Newman black hole," Physical Review D, vol. 41, no. 4, pp. 1086-1090, 1990.

[22] K. S. Virbhadra, "Energy distribution in Kerr-Newman spacetime in Einstein's as well as Møller's prescriptions," Physical Review D, vol. 42, pp. 2919-2921, 1990.

[23] N. Rosen and K. S. Virbhadra, "Energy and momentum of cylindrical gravitational waves," General Relativity and Gravitation, vol. 25, no. 4, pp. 429-433, 1993. 
[24] J. M. Aguirregabiria, A. Chamorro, and K. S. Virbhadra, "Energy and angular momentum of charged rotating black holes," General Relativity and Gravitation, vol. 28, no. 11, pp. 1393-1400, 1996.

[25] K. S. Virbhadra, "Naked singularities and Seifert's conjecture," Physical Review D, vol. 60, no. 10, Article ID 104041, 6 pages, 1999.

[26] S. S. Xulu, "Bergmann-Thomson energy-momentum complex for solutions more general than the Kerr-Schild class," International Journal of Theoretical Physics, vol. 46, no. 11, pp. 29152922, 2007.

[27] S. S. Xulu, "Møller energy for the Kerr-Newman metric," Modern Physics Letters A, vol. 15, no. 24, pp. 1511-1517, 2000.

[28] I.-C. Yang and I. Radinschi, "On the difference of energy between the Einstein and Møller prescription," Chinese Journal of Physics, vol. 42, no. 1, pp. 40-44, 2004.

[29] I. Radinschi and T. Grammenos, “Møller's energy-momentum complex for a spacetime geometry on a noncommutative curved D3-brane," International Journal of Theoretical Physics, vol. 47, no. 5, pp. 1363-1372, 2008.

[30] I. Radinschi, F. Rahaman, and A. Ghosh, "On the energy of charged black holes in generalized dilaton-axion gravity," International Journal of Theoretical Physics, vol. 49, no. 5, pp. 943-956, 2010.

[31] I. Radinschi, F. Rahaman, and A. Banerjee, "On the energy of Hořava-Lifshitz black holes," International Journal of Theoretical Physics, vol. 50, no. 9, pp. 2906-2916, 2011.

[32] R. M. Gad, "Energy and momentum densities of cosmological models, with equation of state $\rho=\mu$, in general relativity and teleparallel gravity," International Journal of Theoretical Physics, vol. 46, pp. 3263-3274, 2007.

[33] M. Abdel-Megied and R. M. Gad, "Møller's energy in the Kantowski-Sachs space-time," Advances in High Energy Physics, vol. 2010, Article ID 379473, 6 pages, 2010.

[34] R. M. Gad, "On teleparallel version of stationary axisymmetric solutions and their energy contents," Astrophysics and Space Science, vol. 346, no. 2, pp. 553-557, 2013.

[35] T. Bringley, "Energy and momentum of a stationary beam of light," Modern Physics Letters A: Particles and Fields, Gravitation, Cosmology, Nuclear Physics, vol. 17, no. 3, pp. 157-161, 2002.

[36] M. Sukenik and J. Sima, "Energy and momentum in expansive nondecelerative universe," http://arxiv.org/abs/gr-qc/0101026.

[37] M. Sharif and T. Fatima, "Energy distribution associated with static axisymmetric solutions," Astrophysics and Space Science, vol. 302, no. 1-4, pp. 217-224, 2006.

[38] M. Sharif and M. Azam, "Energy-momentum distribution: some examples," International Journal of Modern Physics A, vol. 22, no. 10, pp. 1935-1951, 2007.

[39] P. Halpern, "Energy of the Taub cosmological solution," Astrophysics and Space Science, vol. 306, no. 4, pp. 279-283, 2006.

[40] E. C. Vagenas, "Energy distribution in 2D stringy black hole backgrounds," International Journal of Modern Physics A, vol. 18, no. 31, pp. 5781-5794, 2003.

[41] E. C. Vagenas, "Effective mass of radiating charged particle in Einstein's universe," Modern Physics Letters A: Particles and Fields, Gravitation, Cosmology, Nuclear Physics, vol. 19, no. 3, pp. 213-222, 2004.

[42] E. C. Vagenas, "Energy distribution in a BTZ black hole spacetime," International Journal of Modern Physics D, vol. 14, no. 3-4, pp. 573-586, 2005.
[43] E. C. Vagenas, "Energy distribution in the dyadosphere of a Reissner-Nordström black hole in Møller's prescription," Modern Physics Letters A, vol. 21, no. 25, pp. 1947-1956, 2006.

[44] T. Multamäki, A. Putaja, E. C. Vagenas, and I. Vilja, "Energymomentum complexes in $f(R)$ theories of gravity," Classical and Quantum Gravity, vol. 25, no. 7, Article ID 075017, 2008.

[45] L. Balart, "Energy distribution of (2+1)-dimensional black holes with nonlinear electrodynamics," Modern Physics Letters A, vol. 24, no. 34, pp. 2777-2785, 2009.

[46] A. M. Abbassi, S. Mirshekari, and A. H. Abbassi, "Energymomentum distribution in static and nonstatic cosmic string space-times," Physical Review D, vol. 78, no. 6, Article ID 064053, 9 pages, 2008.

[47] J. Matyjasek, "Some remarks on the Einstein and Møller pseudotensors for static and spherically-symmetric configurations," Modern Physics Letters A, vol. 23, no. 8, pp. 591-601, 2008.

[48] S. K. Tripathy, B. Mishra, G. K. Pandey, A. K. Singh, T. Kumar, and S. S. Xulu, "Energy and momentum of Bianchi type VI_h universes," http://arxiv.org/abs/1501.04990.

[49] C. Møller, "The four-momentum of an insular system in general relativity," Nuclear Physics, vol. 57, pp. 330-338, 1964.

[50] K. Hayashi and T. Shirafuji, "New general relativity," Physical Review D: Particles and Fields, vol. 19, no. 12, pp. 3524-3553, 1979.

[51] J. M. Nester, L. L. So, and T. Vargas, "Energy of homogeneous cosmologies," Physical Review D: Particles, Fields, Gravitation, and Cosmology, vol. 78, no. 4, Article ID 044035, 11 pages, 2008.

[52] G. G. Nashed and T. Shirafuji, "Reissner-Nordström spacetime in the tetrad theory of gravitation," International Journal of Modern Physics D: Gravitation, Astrophysics, Cosmology, vol. 16, no. 1, pp. 65-79, 2007.

[53] G. G. L. Nashed, "Møller's energy of Kerr-NUT metric," Chinese Physics Letters, vol. 25, no. 4, pp. 1202-1204, 2008.

[54] J. W. Maluf, F. F. Faria, and S. C. Ulhoa, "On reference frames in spacetime and gravitational energy in freely falling frames," Classical and Quantum Gravity, vol. 24, no. 10, pp. 2743-2753, 2007.

[55] J. W. Maluf, M. V. O. Veiga, and J. F. da Rocha-Neto, "Regularized expression for the gravitational energy-momentum in teleparallel gravity and the principle of equivalence," General Relativity and Gravitation, vol. 39, no. 3, pp. 227-240, 2007.

[56] A. A. Sousa, R. B. Pereira, and A. C. Silva, "Energy and angular momentum densities in a Gödel-type universe in teleparallel geometry," Gravitation and Cosmology, vol. 16, no. 1, pp. 25-33, 2010.

[57] M. Sharif and S. Taj, "Energy contents of some non-vacuum spacetimes in teleparallel gravity," Astrophysics and Space Science, vol. 325, no. 1, pp. 75-83, 2009.

[58] Y.-X. Liu, Z.-H. Zhao, J. Yang, and Y.-S. Duan, "The total energy-momentum of the universe in teleparallel gravity," http://arxiv.org/abs/0706.3245.

[59] G. G. L. Nashed, "Kerr-Newman solution and energy in teleparallel equivalent of Einstein theory," Modern Physics Letters A, vol. 22, no. 14, pp. 1047-1056, 2007.

[60] G. G. L. Nashed, “Møller's Energy of Kerr-NUT Metric," Chinese Physics Letters, vol. 25, no. 4, pp. 1202-1204, 2008.

[61] G. G. L. Nashed, "Energy and angular momentum of general four-dimensional stationary axi-symmetric space-time in teleparallel geometry," International Journal of Modern Physics A, vol. 23, no. 12, pp. 1903-1918, 2008.

[62] G. G. L. Nashed, "Brane world black holes in teleparallel theory equivalent to general relativity and their Killing vectors, energy, 
momentum and angular momentum," Chinese Physics B, vol. 19, no. 2, Article ID 020401, 2010.

[63] M. Sharif and A. Jawad, "Energy contents of some well-known solutions in teleparallel gravity," Astrophysics and Space Science, vol. 331, no. 1, pp. 257-263, 2010.

[64] C.-C. Chang, J. M. Nester, and C.-M. Chen, "Pseudotensors and quasilocal energy-momentum," Physical Review Letters, vol. 83, no. 10, pp. 1897-1901, 1999.

[65] J. M. Nester, C.-M. Chen, J.-L. Liu, and G. Sun, "A reference for the covariant hamiltonian boundary term," in Relativity and Gravitation-100 Years After Einstein in Prague, J. Bicak and T. Ledvinka, Eds., vol. 157 of Springer Proceedings in Physics, pp. 177-184, Springer, Cham, Switzerland, 2014.

[66] K. S. Virbhadra and C. R. Keeton, "Time delay and magnification centroid due to gravitational lensing by black holes and naked singularities," Physical Review D, vol. 77, Article ID 124014, 2008.

[67] P. K. Sahoo, K. L. Mahanta, D. Goit et al., "Einstein energymomentum complex for a phantom black hole metric," Chinese Physics Letters, vol. 32, no. 2, Article ID 020402, 2015.

[68] J. P. DeAndrea and K. M. Alexander, "Negative time delay in strongly naked singularity lensing," Physical Review D, vol. 89, Article ID 123012, 2014.

[69] A. K. Sinha, G. K. Pandey, A. K. Bhaskar et al., "Effective gravitational mass of the Ayón-Beato and García metric," Modern Physics Letters A, http://arxiv.org/abs/1501.01615.

[70] J. M. Bardeen, "Non-singular general-relativistic gravitational collapse," in Proceedings of the International Conference GR5, p. 174, USSR, Tbilisi, Georgia, 1968.

[71] S. Ansoldi, "Spherical black holes with regular center: a review of existing models including a recent realization with Gaussian sources," in Proceedings of the Conference on Black Holes and Naked Singularities, Milan, Italy, May 2007, http://arxiv.org/abs/0802.0330.

[72] B. Hoffmann and L. Infeld, "On the choice of the action function in the new field theory," Physical Review, vol. 51, no. 9, pp. 765773, 1937.

[73] E. Ayón-Beato and A. García, "Regular black hole in general relativity coupled to nonlinear electrodynamics," Physical Review Letters, vol. 80, no. 23, pp. 5056-5059, 1998.

[74] E. Ayón-Beato and A. García, "New regular black hole solution from nonlinear electrodynamics," Physics Letters B, vol. 464, no. 1-2, pp. 25-29, 1999.

[75] E. Ayón-Beato and A. García, "Non-singular charged black hole solution for non-linear source," General Relativity and Gravitation, vol. 31, no. 5, pp. 629-633, 1999.

[76] M. Cataldo and A. García, "Regular (2+1)-dimensional black holes within nonlinear electrodynamics," Physical Review D, vol. 61, Article ID 084003, 2000.

[77] K. A. Bronnikov, "Regular magnetic black holes and monopoles from nonlinear electrodynamics," Physical Review D, vol. 63, no. 4, Article ID 044005, 2001.

[78] A. Burinskii and S. R. Hildebrandt, "New type of regular black holes and particlelike solutions from nonlinear electrodynamics," Physical Review D, vol. 65, no. 10, Article ID 104017, 2002.

[79] J. Matyjasek, "Extremal limit of the regular charged black holes in nonlinear electrodynamics," Physical Review D, vol. 70, no. 4, Article ID 047504, 2004.

[80] E. Ayón-Beato and A. García, "Four-parametric regular black hole solution," General Relativity and Gravitation, vol. 37, no. 4, pp. 635-641, 2005.
[81] I.-C. Yang, C.-L. Lin, and I. Radinschi, “The energy of regular black hole in general relativity coupled to nonlinear electrodynamics," International Journal of Theoretical Physics, vol. 48, no. 1, pp. 248-255, 2009.

[82] J. Matyjasek, D. Tryniecki, and M. Klimek, "Regular black holes in an asymptotically de Sitter universe," Modern Physics Letters A, vol. 23, pp. 3377-3392, 2009.

[83] I. Dymnikova, "Regular electrically charged vacuum structures with de Sitter centre in nonlinear electrodynamics coupled to general relativity," Classical and Quantum Gravity, vol. 21, no. 18, pp. 4417-4428, 2004.

[84] S. H. Hendi, "Asymptotic Reissner-Nordström black holes," Annals of Physics, vol. 333, pp. 282-289, 2013.

[85] E. Ayón-Beato and A. García, "The Bardeen model as a nonlinear magnetic monopole," Physics Letters B, vol. 493, no. 1-2, pp. 149-152, 2000.

[86] L. Balart and E. C. Vagenas, "Regular black hole metrics and the weak energy condition," Physics Letters. B, vol. 730, pp. 14-17, 2014.

[87] L. Balart and E. C. Vagenas, "Regular black holes with a nonlinear electrodynamics source," Physical Review D, vol. 90, Article ID 124045, 2014.

[88] S. A. Hayward, "Formation and evaporation of nonsingular black holes," Physical Review Letters, vol. 96, Article ID 031103, 2006.

[89] J. M. Cohen and F. de Felice, "The total effective mass of the Kerr-Newman metric," Journal of Mathematical Physics, vol. 25, no. 4, pp. 992-994, 1984.

[90] S. Nojiri and S. D. Odintsov, "Instabilities and anti-evaporation of Reissner-Nordström black holes in modified $F(R)$ gravity," Physics Letters B, vol. 735, pp. 376-382, 2014. 


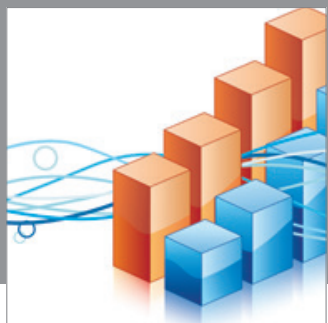

Advances in

Operations Research

mansans

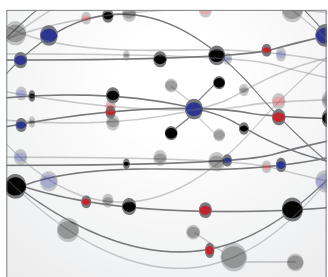

The Scientific World Journal
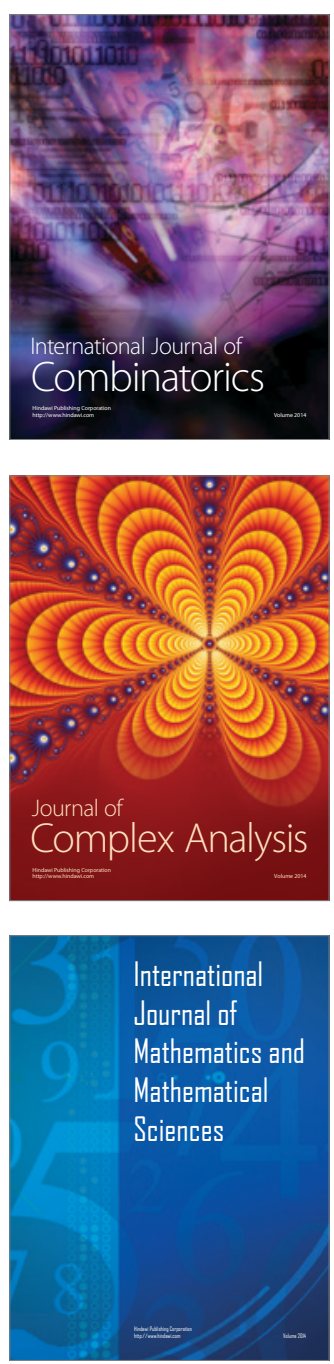
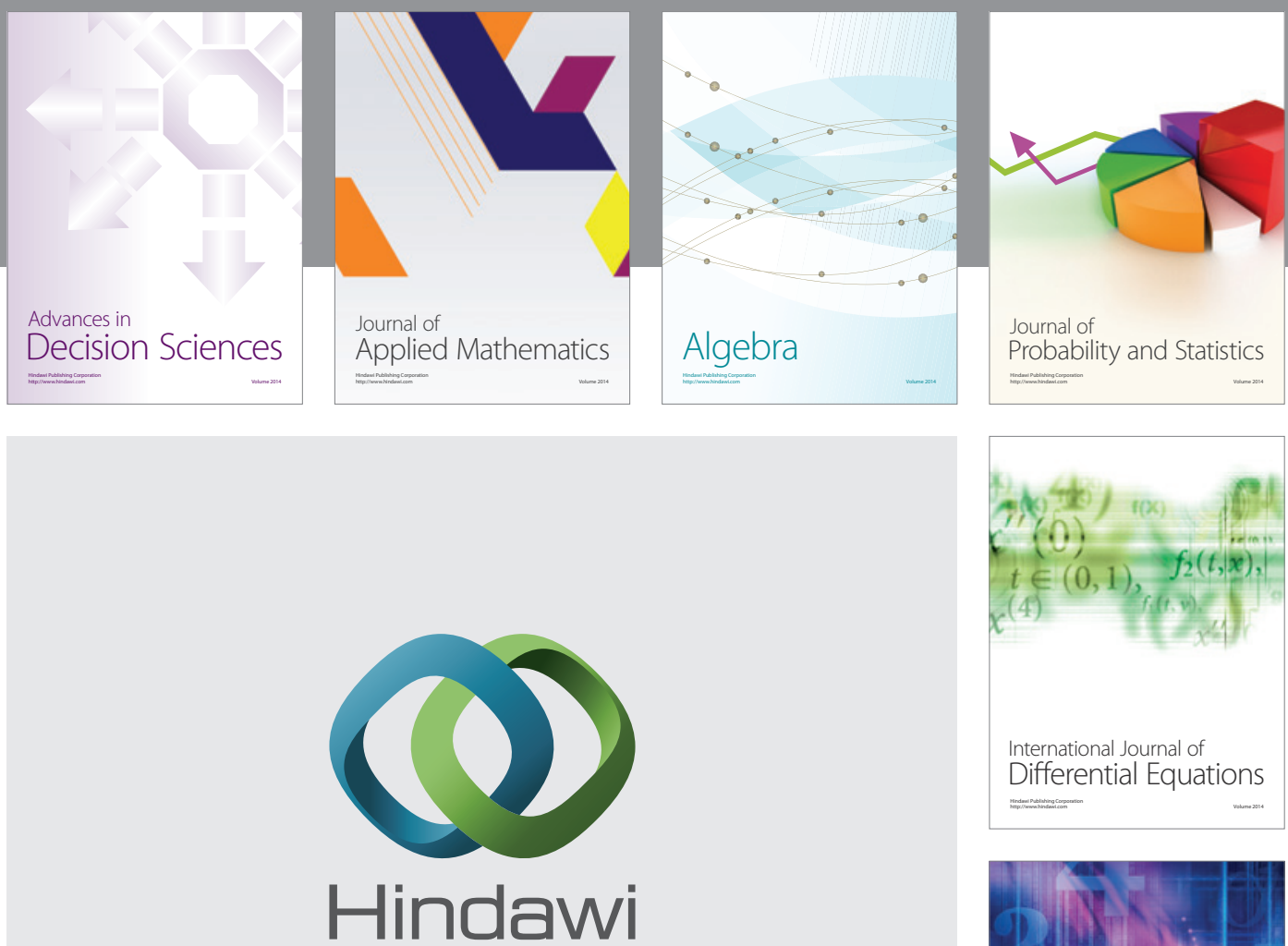

Submit your manuscripts at http://www.hindawi.com
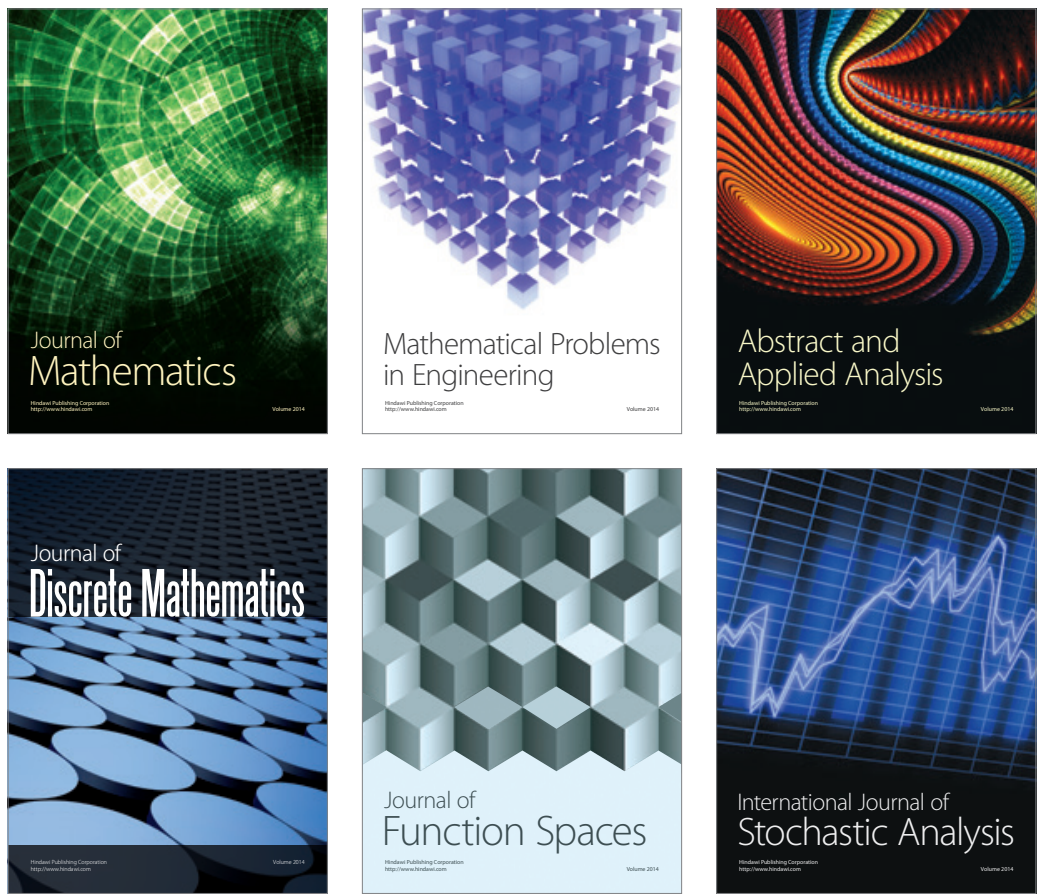

Journal of

Function Spaces

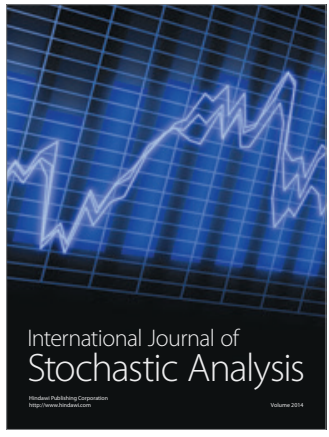

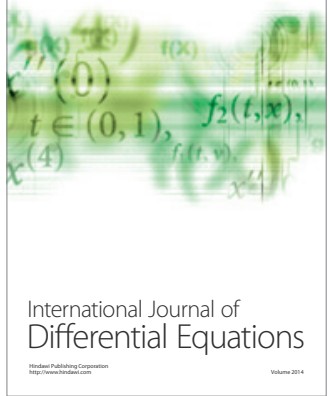
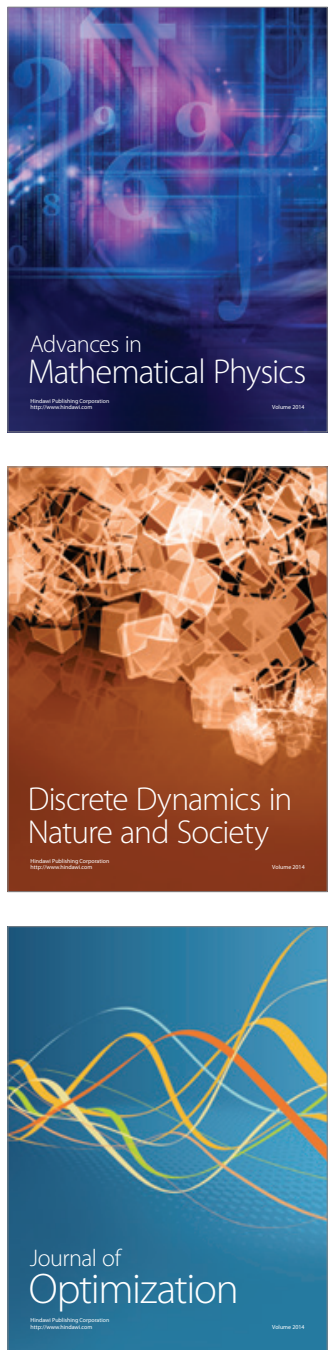